\title{
Solution of an Initial Value Problemin Ordinary Differential Equations Using the Quadrature Algorithm Based on the Heronian Mean
}

\author{
Bazuaye Frank Etin-Osa \\ Department of Mathematics and Statistics, University of Port Harcourt, Port Harcourt, Rivers State, Nigeria \\ Email address: \\ febazuaye@yahoo.com
}

\section{To cite this article:}

Bazuaye Frank Etin-Osa. Solution of an Initial Value Problemin Ordinary Differential Equations Using the Quadrature Algorithm Based on the Heronian Mean. International Journal of Applied Mathematics and Theoretical Physics. Vol. 5, No. 2, 2019, pp. 45-51.

doi: $10.11648 / j$. .jamtp.20190502.12

Received: May 16, 2019; Accepted: June 13, 2019; Published: August 6, 2019

\begin{abstract}
Over the years, the Quadrature Algorithm as a method of solving initial value problems in ordinary differential equations is known to be of low accuracy compared to other well known methods. However, It has been shown that the method perform well when applied to moderately stiff problems. In this present study, the nonlinear method based on the Heronian Mean (HeM), of the function value for the solution of initial value problems is developed. Stability investigation is in agreement with the known Trapezoidal method.
\end{abstract}

Keywords: Harmonic Mean, Stability, Stiff Problems, Geometric Mean, Accuracy

\section{Introduction}

Ordinary differential equations are the major form of mathematical model occurring in Science and Engineering, and, consequently, the numerical solution of differential equations is a very large area of research. The advent of computers has tremendously revolutionized the type and variety of numerical methods over the past three decades and these methods are applied to solve mathematical problems. Several methods have been developed using the idea of different means such as the geometric mean, centroidal mean, harmonic mean, contra-harmonic mean and the heronian mean. The three stage method based on the harmonic mean and a multi-derivative method using the usual arithmetic mean were developed respectively [1] and [2]. Also, a thirdorder method based on the geometric mean was presented [3]. But a fourth-order method based on the harmonic mean was introduce [4] while the fourth-order method which is an embedded method based on the arithmetic and harmonic mean was constructed [5] The comparison of modified Runge-Kutta methods based on varieties of means was studied [6]. The New 4TH Order Hybrid Runge -kutta methods for solving Ivps in ODEs was investigated [7]. In the paper, a new 4th Order Hybrid Runge-Kutta method based on linear combination of Arithmetic mean, Geometric mean and the Harmonic mean to solve first order initial value problems (IVPs) in ordinary differential equations (ODEs).

The trapezoidal rule for the numerical integration of firstorder ordinary differential equations is shown to possess, for a certain type of problem, an undesirable property. The removal of this difficulty is shown to be straightforward, resulting in a modified trapezoidal rule. Whilst this latent difficulty is slight (and probably rare in practice), the fact that the proposed modification involves negligible additional programming effort would suggest that it is worthwhile. A corresponding modification for the trapezoidal rule for the Goursat problem is also included.[8]presented a nonlinear trapezoidal formula for the solution of the Goursat problem. The new scheme implements the harmonic mean (HM) averaging of the functional values rather than the arithmetic mean (AM) or the geometric mean (GM) averaging. A comparison is made with the existing techniques, and the results obtained show better approximations related to the accuracy level in favor of the HM strategy. [9] considereda Third Runge-Kutta Method based on a Linear combination of Arithmetic mean, harmonic mean and geometric mean..[10] derived theRunge-Kutta methods based on averages other than the arithmetic mean is on the rise. In this paper, the 
authors propose a new version of explicit Runge-Kutta method, by introducing the harmonic mean as against the usual arithmetic averages in standard Runge-Kutta schemes.

The a nonlinear mid-point rule formula based on geometric means (GM) for the numerical solution of differential equations $y^{\prime}=f(x, y)$ was presented [11] with supporting numerical results. However, the New fifth order weighted Runge -kutta methods based on the Heronian mean for initial value problems in ordinary differential equations was developed and implemented [12]. In the paper Comparisons in terms of numerical accuracy and size of the stability region between new proposed Runge-Kutta(5,5) algorithm, RungeKutta $(5,5)$ based on Harmonic Mean, Runge-Kutta(5,5) based on Contra Harmonic Mean and Runge-Kutta(5,5) based on Geometric Mean where also investigated.

We consider the numerical solution of the initial value problems.

$$
v^{\prime}=f(u, v) \quad y\left(x_{0}\right)=v_{0}
$$

In the region of $x_{0} \leq x \leq X$.

Numerical integration as the process of finding the value of a definiteintegral,

$$
I=\int_{a}^{b} f(u) d u
$$

With $a \leq u \leq b$ An approximate value of the integral is obtained by replacingthe function by an interpolating polynomial. Thus, different formulas for numericalintegration

would result for different interpolating formulas. In this case, the Newton's forward difference formula shall be applied.

The interval $[a, b]$ is dividedinto nequal subintervals: $a=u_{0}<u_{1}<u_{2}<\ldots u_{n}=b$.

With $u_{n+1}-u_{n}=h$ then (2) becomes:

$$
I=\int_{u_{0}}^{u_{n}} f(u) d u
$$

Suppose $u=u_{0}+r h$ and $d u=h d r u_{n}=u_{0}+n h$

Makinga change of variable from $u$ to $r: u=u_{0}+r h$

The integral(3) becomes,

$$
I=\int_{u_{0}}^{u_{0}+n h} f\left(u_{0}+r h\right) h d r=h \int_{0}^{n} f\left(u_{0}+r h\right) d r
$$

Approximating $f(u)=f\left(u_{0}+r h\right)$ by the Newton's forward difference formula.

Then, from equation (4), and setting $v=f(u)$ :

$$
I=h\left[\int_{0}^{n} u_{0}+r \Delta u_{0}+\frac{r(r-1)}{2} \Delta^{2} u_{0} \frac{r(r-1)(q-2)}{6} \Delta^{3} u_{0}+\ldots\right] d r
$$

Integrating (5) and substituting the limit of integration gives:

$$
\begin{aligned}
& I=h\left[\int_{0}^{n} u_{0}+r \Delta u_{0}+\frac{r(r-1)}{2} \Delta^{2} u_{0} \frac{r(r-1)(q-2)}{6} \Delta^{3} u_{0}+\ldots\right] d r \\
& =n h\left[u_{0}+\frac{n}{2} \Delta u_{0}+\frac{n(2 n-3)}{12} \Delta^{2} u_{0}+\frac{n(n-2)^{2}}{24} \Delta^{3} u_{0}+\left(\frac{n^{4}}{5}-\frac{3 n^{3}}{2}+\frac{11 n^{2}}{3}-3 n\right) \frac{\Delta^{4} u_{0}}{4 !}+\ldots\right]
\end{aligned}
$$

One of the methods in literature for solving (1) is the trapezoidal rule. This is obtained by setting $n$ equal to 1 , and takes the curve between two consecutive points as linear. Thus, we terminate the sequence on the right in equation (6) at the linear term as the higher difference terms ( $\Delta^{2} u_{0}, \Delta^{3} u_{0}$, ) etcwould be zero. Then,

The Trapezoidal rule for the solution of (1) is given as:

$$
y_{n+1}=y_{n}+\frac{h}{2}\left[f\left(u_{n}, v_{n}\right)+f\left(u_{n+1}, v_{n+1}\right)\right]
$$

where $\mathrm{h}$ is the mesh length in the $\mathrm{u}$ direction.

\section{Materials and Methods}

The numerical algorithm (7) is a one-step implicit method that has the desirable features of performing well when applied to stiff problems and also being A-stable method [13]. In another approach [14], the nonlinear equivalent of the trapezoidal formula (7), referred to as the geometric mean(GM) Euler formula was derived [13]. The result reveals that for certain function $\lambda(x)$, the stability requirement imposes certain

$$
\left.h\left[\lambda\left(x_{n}\right)\right]-\lambda\left(x_{n+1}\right)\right] \leq 4
$$

restrict the step length to lie in the interval:

$$
0<h \leq 4\left[\lambda\left(x_{n}\right)-\lambda\left(x_{n+1}\right)\right]^{-1}
$$

This is a big challenge.[8]developed an alternative strategy to circumvent this challenge by replacing (1) by:

$$
y_{n+1}=y_{n}+\frac{h}{2}\left[f\left(u_{n+\frac{1}{2}}, v_{n}\right)+f\left(u_{n+\frac{1}{2}}, v_{n+1}\right)\right]
$$

Similarly the approach [8], the effect of the nonlinear formula based on the geometric mean instead of the 
arithmetic means (AM) in the trapezoidal formula (1). was demonstrated [14] Instead of (7) they considered the geometric mean (GM) formula was

$$
\begin{gathered}
y_{n+1}=y_{n}+h \sqrt{f\left(u_{n}\right) f\left(u_{n+1}\right)} \quad \text { (8) Mean (Mean) as: } \\
v_{n+1}=v_{n}+\frac{h}{3}\left[f\left(u_{n}, v_{n}\right)+f\left(u_{n+1}, v_{n+1}\right)+\sqrt{f\left(u_{n}, v_{n}\right) f\left(u_{n+1}, v_{n+1}\right)}\right]
\end{gathered}
$$

Due to the modification to [8], it gives a better accuracy when applied to certain problems.

In this present paper, the Arithmetic mean (AM) (7) and the Geometric Mean (GM) in (8)arereplaced by the Heronian

The method (9) above is implicit with high order.

It is truly preferable to write (9) as:

$$
v_{n+1}^{(i+1)}=v_{n}+\frac{h}{3}\left[f\left(u_{n}, v_{n}\right)+f\left(u_{n+1}, v_{n+1}^{(i)}\right)+\sqrt{f\left(u_{n}, v_{n}\right) f\left(u_{n+1}, v^{(i)}{ }_{n+1}\right)}\right]
$$

The starting value $v_{0}^{(1)}$ is obtained by an implicit formula, e.g., the Euler formula. Thus, Solving for $v_{n+1}$ in:

$$
v_{n+1}=v_{n}+\frac{h}{3}\left[f\left(u_{n}, v_{n}\right)+f\left(u_{n+1}, v_{n+1}\right)+\sqrt{f\left(u_{n}, v_{n}\right) f\left(u_{n+1}, v_{n+1}\right)}\right]
$$

Define:

$$
v_{n+1}^{(i+1)}=v_{n}+\frac{h}{3}\left[f\left(u_{n}, v_{n}\right)+f\left(u_{n+1}, v^{(i)}{ }_{n+1}\right)+\sqrt{f\left(u_{n}, v_{n}\right) f\left(u_{n+1}, v^{(i)}{ }_{n+1}\right)}\right]
$$

the scheme would look like (for $i=0$ ),

$$
\begin{gathered}
(i=0), \quad v_{1}^{1}=v_{0}+\frac{h}{3}\left[f\left(u_{0}, v_{0}\right)+f\left(u_{1}, v_{1}^{0}\right)+\sqrt{f\left(u_{0}, v_{0}\right\} f\left(u_{0}, v_{1}^{0}\right)}\right] \\
(i=1), \quad v_{1}^{2}=v_{0}+\frac{h}{3}\left[f\left(u_{0}, v_{0}\right)+f\left(u_{1}, v_{1}^{1}\right)+\sqrt{f\left(u_{0}, v_{0}\right\} f\left(u_{0}, v_{1}^{1}\right)}\right]
\end{gathered}
$$

This is continuing recursively until convergence is obtained.

\section{Results}

Stability and Convergence analysis of the New Scheme.

In this session, the stability of (10) shall be investigated. The stability region largely depends on the initial value problem (IVP). According to [15] and[16], it should be noted that The condition $\left|\frac{u_{n+1}}{u_{n}}\right|<1$ must be satisfied in order to determine the stability region [15] and [16]. To determine the stability region. To study the stability properties of method (11), we apply the new algorithm to the test equation $v^{\prime}=\lambda v$. This will yield:

$$
\frac{v_{n+1}}{v_{n}}=1+\frac{\lambda h}{3}\left[1+\frac{v_{n+1}}{v_{n}}+\sqrt{\frac{v_{n+1}}{v_{n}}}\right]
$$

Defining $\frac{v_{n+1}}{v_{n}}=P_{n}=Z_{n}^{2}$ We obtain:

$$
Z_{n}^{2}=1+\frac{\lambda h}{3}\left[1+Z_{n}^{2}+Z_{n}\right]
$$

Simplifying (13) yields:

$$
\begin{aligned}
& 3 Z_{n}^{2}-\lambda h Z_{n}^{2}-\lambda h Z_{n}-\lambda h-3=0 \\
& Z_{n}^{2}-\left(\frac{\lambda h}{3-\lambda}\right) Z_{n}-\left(\frac{\lambda h+3}{3-\lambda}\right)=0
\end{aligned}
$$

For absolute stability, it is required that the roots:

$$
\left|Z_{n}\right|<1
$$

(14) can be solved to obtain:

$$
\begin{gathered}
Z_{n}=\frac{\frac{\lambda h}{3-\lambda} \pm \sqrt{\left(\frac{\lambda h}{3-\lambda}\right)^{2}-4\left(\frac{\lambda h+3}{3-\lambda}\right)}}{2} \\
{\left[\frac{\lambda h}{6-2 \lambda} \pm \sqrt{\left(\frac{\lambda h}{12-4 \lambda}\right)^{2}-\left(\frac{\lambda h+3}{12-4 \lambda}\right)}\right] \leq 1}
\end{gathered}
$$

(16) is satisfied if: 


$$
\sqrt{\left(\frac{\lambda h}{12-4 \lambda}\right)^{2}-\left(\frac{\lambda h+3}{12-4 \lambda}\right)}<0
$$

This condition is satisfied if $\lambda(u)$ a negative function, which agrees with the present circumstance. There is no restriction on the meshsize as far as the solution of $v^{\prime}=\lambda(u) v$ is the object of consideration by using the present algorithm unlike in the conventional trapezoidal rule.

\section{Discussion}

In this section, we Illustrate the efficiency and suitability of the new computational methods discussed in this paper. The problems can be evaluated with different step sizes.

Problem 1. $v^{\prime}=\frac{1}{v} v(0)=1$ and the exact solution is $u=(2 u+1)^{\frac{1}{2}}$ on $[0,1]$

The results of problem 1 with different values of step sizes are presented in the figures below.

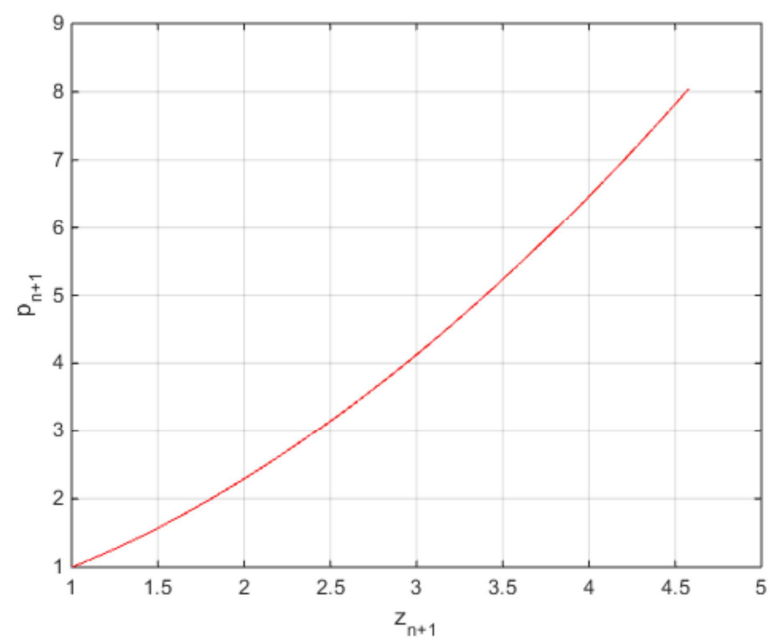

Figure 1. The graph of $p_{n+1}$ against $z_{n+1}$ for $h=0.1$.

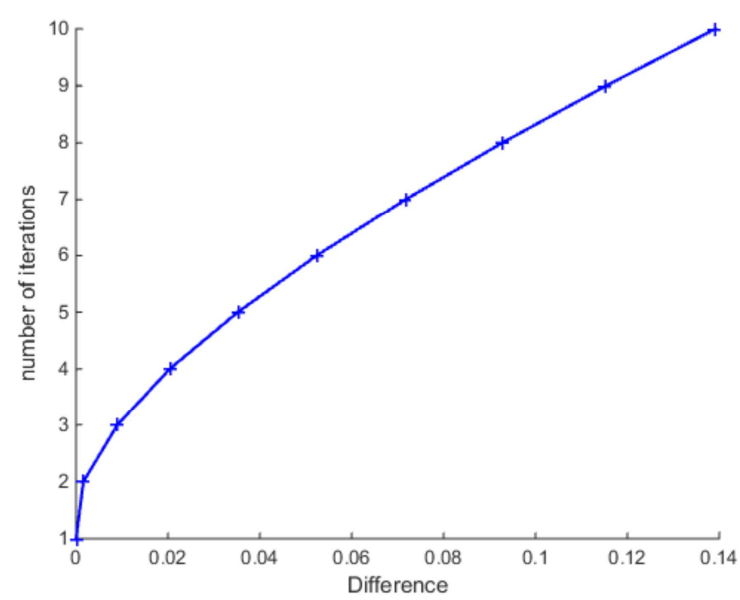

Figure 2. The graph of the number of iterations against $\left|y_{n+1}^{(i+1)}-y_{n+1}^{(i}\right|$ for $h=0.1$.

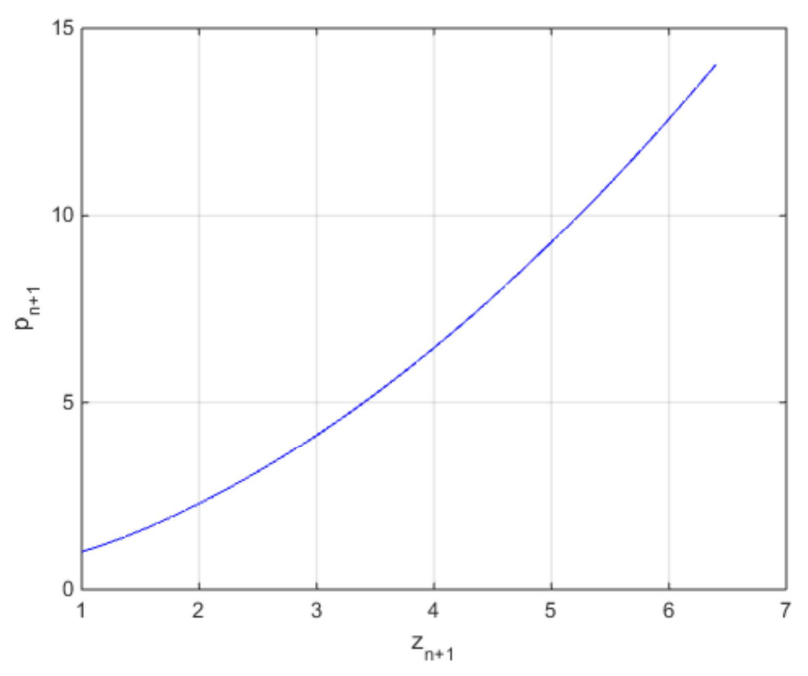

Figure 3. The graph of $p_{n+1}$ against $z_{n+1}$ for $h=0.2$.

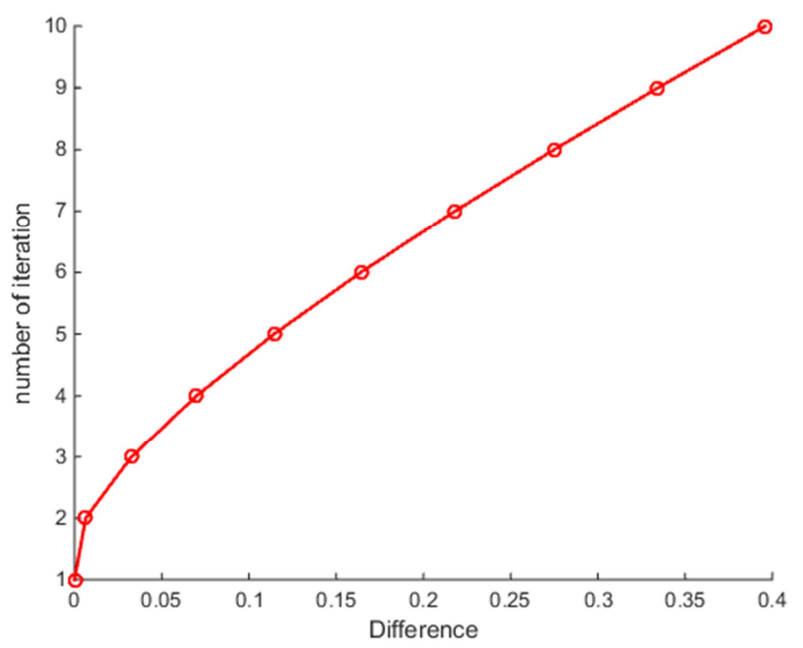

Figure 4. The graph of the number of iterationsagainst $\left|y_{n+1}^{(i+1)}-y_{n+1}^{(i}\right|$ for $h=0.2$.

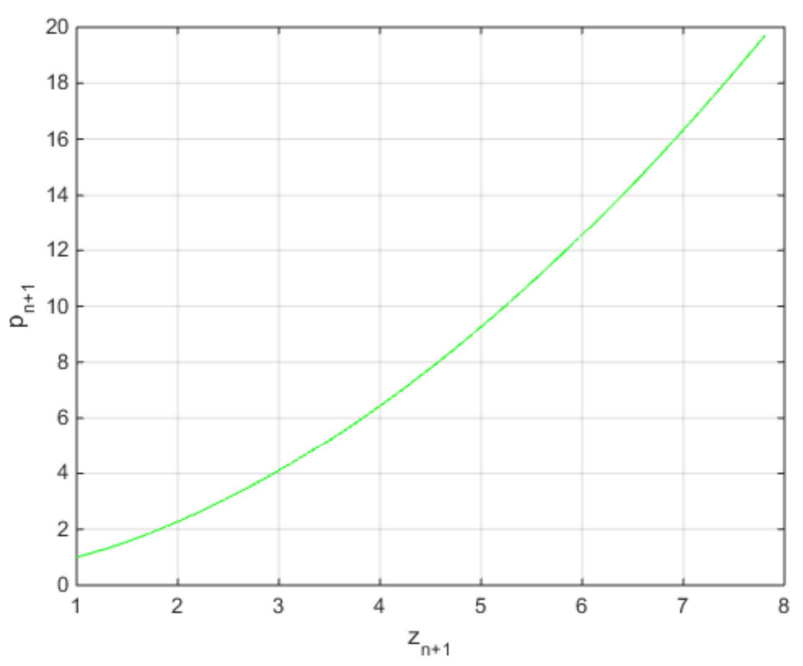

Figure 5. The graph of $p_{n+1}$ against $z_{n+1}$ for $h=0.3$. 


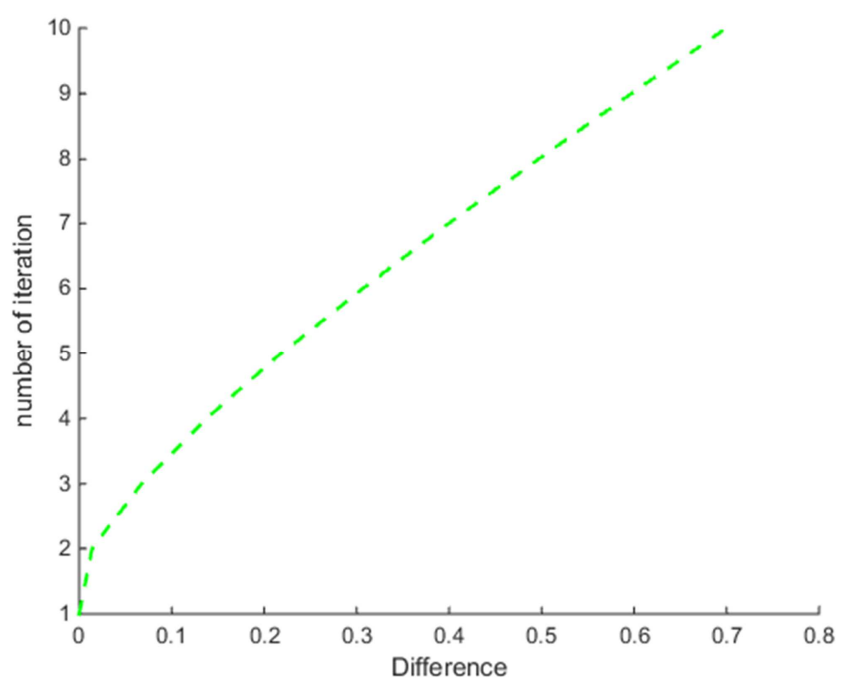

Figure 6. The graph of the number of iterations against $\left|y_{n+1}^{(i+1)}-y_{n+1}^{(i}\right|$ for $h=0.3$.

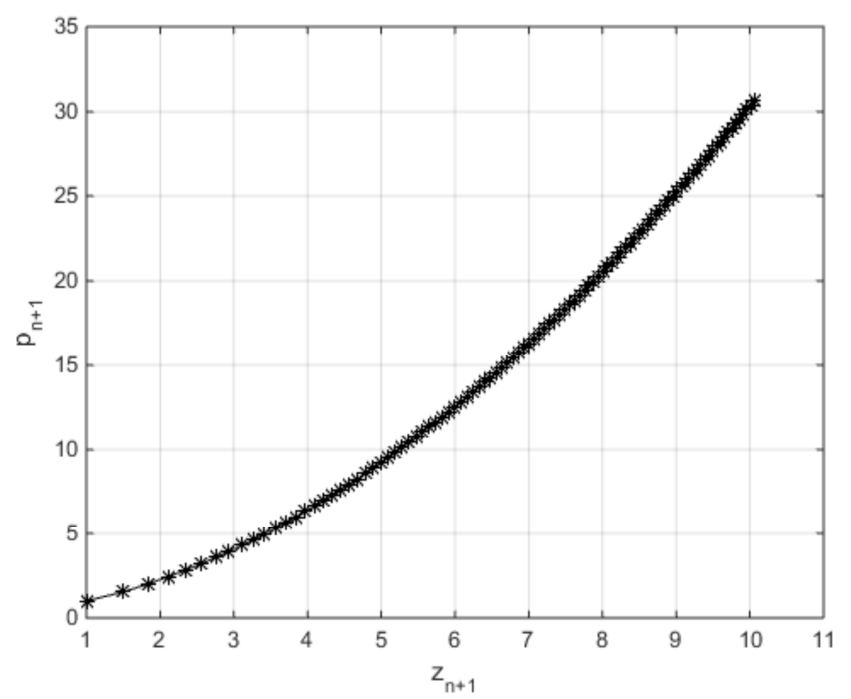

Figure 7. The graph of $p_{n+1}$ against $z_{n+1}$ for $h=0.5$.

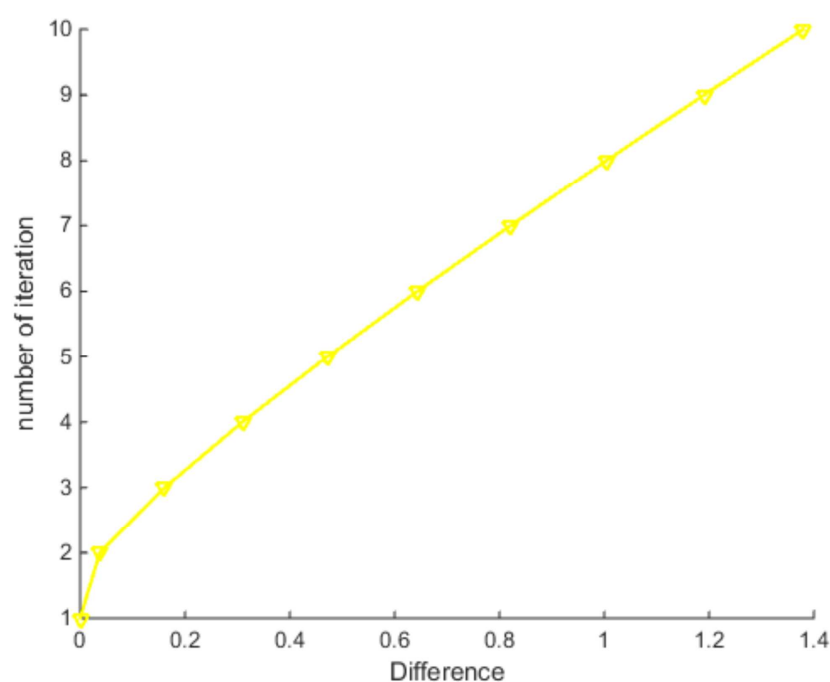

Figure 8. The graph of the number of iterations against $\left|y_{n+1}^{(i+1)}-y_{n+1}^{(i}\right|$ for $h=0.5$.

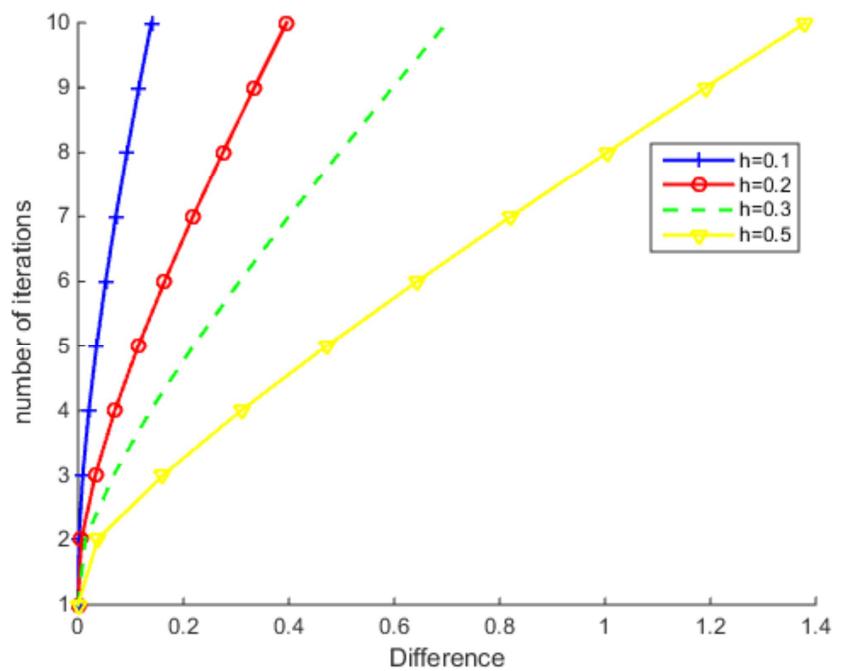

Figure 9. The graph of the number of iterations against $\left|y_{n+1}^{(i+1)}-y_{n+1}^{(i}\right|$ for $h=0.1,0.2,0.3,0.5$.

Table 1. Table of the value of $\left|y_{n+1}^{(i+1)}-y_{n+1}^{(i)}\right|$ for $h=0.1$ for ten iterations.

\begin{tabular}{llll}
\hline $\mathbf{n}$ & $\boldsymbol{y}_{\boldsymbol{n}+1}^{(i)}$ & $\boldsymbol{y}_{\boldsymbol{n}+1}^{(i+1)}$ & $\left|\boldsymbol{y}_{\boldsymbol{n}+1}^{(\boldsymbol{i}+1)}-\boldsymbol{y}_{\boldsymbol{n}+1}^{(\boldsymbol{i}}\right|$ \\
\hline 1 & 1 & 1 & 0.0000 \\
2 & 1.1000 & 1.1016 & 0.0016 \\
3 & 1.1909 & 1.1999 & 0.0090 \\
4 & 1.2749 & 1.2953 & 0.0204 \\
5 & 1.3533 & 1.3885 & 0.0352 \\
6 & 1.4272 & 1.4796 & 0.0524 \\
7 & 1.4973 & 1.5690 & 0.0717 \\
8 & 1.5641 & 1.6569 & 0.0928 \\
9 & 1.6280 & 1.7434 & 0.1154 \\
10 & 1.6894 & 1.8286 & 0.1392 \\
\hline
\end{tabular}


Table 2. Table of the value of $\left|y_{n+1}^{(i+1)}-y_{n+1}^{(i)}\right|$ for $h=0.2$ for ten iterations.

\begin{tabular}{llll}
\hline $\mathbf{n}$ & $\boldsymbol{y}_{\boldsymbol{n}+1}^{(\boldsymbol{i})}$ & $\boldsymbol{y}_{\boldsymbol{n}+1}^{(\boldsymbol{i}+1)}$ & $\left|\boldsymbol{y}_{\boldsymbol{n}+1}^{(\boldsymbol{i}+1)}-\boldsymbol{y}_{\boldsymbol{n}+1}^{(\boldsymbol{i}}\right|$ \\
\hline 1 & 1 & 1 & 0.0000 \\
2 & 1.2000 & 1.2064 & 0.0064 \\
3 & 1.3667 & 1.3993 & 0.0326 \\
4 & 1.5130 & 1.5829 & 0.0699 \\
5 & 1.6452 & 1.7596 & 0.1144 \\
6 & 1.7668 & 1.9310 & 0.1642 \\
7 & 1.8800 & 2.0980 & 0.2180 \\
8 & 1.9863 & 2.2613 & 0.2750 \\
9 & 2.0870 & 2.4215 & 0.3345 \\
10 & 2.1829 & 2.5790 & 0.3961 \\
\hline
\end{tabular}

Table 3. Table of the value of $\left|y_{n+1}^{(i+1)}-y_{n+1}^{(i)}\right|$ for $h=0.3$ for ten iterations.

\begin{tabular}{llll}
\hline $\mathbf{n}$ & $\boldsymbol{y}_{\boldsymbol{n}+1}^{(i)}$ & $\boldsymbol{y}_{\boldsymbol{n}+1}^{(i+1)}$ & $\left|\boldsymbol{y}_{\boldsymbol{n}+1}^{(i+1)}-\boldsymbol{y}_{\boldsymbol{n}+1}^{(\boldsymbol{i}}\right|$ \\
\hline 1 & 1 & 1 & 0.0000 \\
2 & 1.3000 & 1.3140 & 0.014 \\
3 & 1.5308 & 1.5981 & 0.0673 \\
4 & 1.7267 & 1.8646 & 0.1379 \\
5 & 1.9005 & 2.1192 & 0.2187 \\
6 & 2.0583 & 2.3649 & 0.3066 \\
7 & 2.2041 & 2.6037 & 0.3996 \\
8 & 2.3402 & 2.8369 & 0.4967 \\
9 & 2.4684 & 3.0655 & 0.5971 \\
10 & 2.5899 & 3.2900 & 0.704 \\
\hline
\end{tabular}

Table4. Table of the value of $\left|y_{n+1}^{(i+1)}-y_{n+1}^{(i)}\right|$ for $h=0.5$ for ten iterations.

\begin{tabular}{llll}
\hline $\mathbf{n}$ & $\boldsymbol{y}_{\boldsymbol{n}+1}^{(\boldsymbol{i})}$ & $\boldsymbol{y}_{\boldsymbol{n}+1}^{(\boldsymbol{i}+1)}$ & $\left|\boldsymbol{y}_{\boldsymbol{n}+1}^{(\boldsymbol{i}+1)}-\boldsymbol{y}_{\boldsymbol{n}+1}^{(\boldsymbol{i}}\right|$ \\
\hline 1 & 1 & 1 & 0.0000 \\
2 & 1.5000 & 1.5375 & 0.0375 \\
3 & 1.8333 & 1.9945 & 0.1612 \\
4 & 2.1061 & 2.4160 & 0.3099 \\
5 & 2.3435 & 2.8158 & 0.4723 \\
6 & 2.5568 & 3.2005 & 0.6437 \\
7 & 2.7524 & 3.5738 & 0.8214 \\
8 & 2.9340 & 3.9381 & 1.0041 \\
9 & 3.1045 & 4.2951 & 1.1906 \\
$\mathrm{n} 10$ & 3.2655 & 4.6459 & 1.3804 \\
\hline
\end{tabular}

\section{Conclusion}

In this paper, the relevance studies in literature were reviewed and the gaps identified. We have also successfully derived the Quadrature Algorithm based on the Harmonic (HM). This method is an improvement over the ones in literature. Also, the stability investigated with the aid of a MATLAB. Practical applicable problems have been considered to test the convergence of the scheme. The results indicate that the New method is stable. The convergence analysis indicate that only a slight increase in computing time is needed in the analysis to give a favorable result.

\section{References}

[1] A. S Wusu, S. A Okunuga, A. B Sofoluwe (2012). A ThirdOrder Harmonic explicit Runge-Kutta Method for Autonomous initial value problems. Global Journal of Pure and Applied Mathematics. 8, 441-451

[2] A. S Wusu, and M. A Akanbi (2013). A Three stage Multiderivative Explicit Rungekutta Method. American Journal of Computational Mathematics. 3, 121-126

[3] M. A Akanbi. (2011). On 3- stage Geometric Explicit RungeKutta Method for singular Autonomous initial value problems in ordinary differential equations Computing.92, 243-263 
[4] J. D Evans and N. B Yaacob (1995). A Fourth Order Runge Kutta Methods based on Heronian Mean Formula. International Journal of Computer Mathematics 58,103-115

[5] N. B Yaacob and B. Sangui (1998). A New Fourth Order Embedded Method based on Heronian Mean Mathematica Jilid, 1998, 1-6.

[6] Wazwaz A. M. (1990). A Modified Third order Runge-kutta Method. Applied Mathematics Letter, 3(1990), 123-125

[7] F. E Bazuaye (2018). A new $4^{\text {th }}$ order Hybrid Runge-Kutta Methods for solving initial value problems. Pure and Applied Mathematics Journal 7(6): 78-87

[8] A. R Gourlay (1970). A note on trapezoidal methods for the solution of initial value problems. Math. Comput.. 24(1), 629633 .

[9] Rini Y. Imran M, Syamsudhuha. (2014). A Third RungeKutta Method based on a Linear combination of Arithmetic mean, harmonic mean and geometric mean. Applied and Computational Mathematics. 2014, 3(5), 231-234

[10] Ashiribo Senapon Wusu1, Moses Adebowale Akanbil, Bakre Omolara Fatimah (2015). On the derivation and implementation of four stage Harmonic Explicit Runge -kutta Method. Applied Mathematics. 6, 694-699
[11] D. J Evans (1988). A Stable Nonlinear Mid-Point Formula for Solving O. D. E. s. Appl. Math. L\&t. Vol. 1, No. 2, pp. 165169.

[12] M. CHANDRU, R. PONALAGUSAMY, P. J. A. ALPHONSE (2017). A New fifth order weighted Runge -kutta methods based on the Heronian mean for initial value problems in ordinary differential equations. J. Appl. Math. \& Informatics Vol. 35 (2017), 191 - 204.

[13] Evans J. D and Sangui B. B (1988). A nonlinear Trapezoidal Formula for the solution of initial value problems. Comput. Math. Applic. 15 (1), 77-79

[14] Evans J. D and Sangui B. B (1991). A Comparison of Numerical O. D. E solvers based on Arithmetic and Geometric means. International Journal of Computer Mthematics, 32-35

[15] S. O. Fatunla (1986). Numerical Treatment of singular IVPs. Computational Math. Application. 12:1109-1115.

[16] J. D Lawson (1966) An order Five R-K Processes with Extended region of Absolute stability. SIAM J. Numer. Anal 4:372-380. 